\title{
Prevalência do transtorno depressivo maior em pessoas com dependência química*
}

Pablo Michel Barcelos Pereira ${ }^{1,2}$

(D) https://orcid.org/0000-0002-9197-8623

Rafael Mariano de Bitencourt ${ }^{1}$

(D) https://orcid.org/0000-0003-4694-3808

Artigo extraído da dissertação de mestrado "Prevalência do Transtorno Depressivo Maior em Pessoas com Dependência Química", apresentada à Universidade do Sul de Santa Catarina, Tubarão, Santa Catarina, Brasil.

${ }^{1}$ Universidade do Sul de Santa Catarina, Laboratório de Neurociência Comportamental, Tubarão, SC, Brasil.

2 Bolsista da Coordenação de Aperfeiçoamento de Pessoal de Nível Superior (CAPES), Brasil.
Objetivo: verificar a prevalência do Transtorno Depressivo Maior em pessoas com dependência química. Método: estudo transversal com a aplicação da entrevista estruturada para os transtornos do DSM-V em 183 indivíduos internados em comunidades terapêuticas de acolhimento para dependentes químicos, localizadas no sul de Santa Catarina, durante o de ano de 2019. Resultados: todos os participantes não realizaram consulta psiquiátrica ao ingressar na instituição e a maioria não realizou consulta psiquiátrica regular (94,5\%). Constatouse que 89 participantes $(55,3 \%)$ possuíam critérios diagnósticos para o Transtorno Depressivo Maior e $59,1 \%$ destes possuíam adicção ao álcool $(p<0,028)$. Conclusão: o diagnóstico do transtorno depressivo deve-se consolidar como uma variável importante para a eficácia do tratamento, visto que sua prevalência é elevada e possui repercussões sobre a qualidade do tratamento e tempo de institucionalização.

Descritores: Transtorno Depressivo Maior; Transtornos Relacionados ao Uso de Substâncias; Epidemiologia; Reabilitação.

\section{Como citar este artigo}

Pereira PMB, Bitencourt RM. Prevalence of major depressive disorder in people with chemical addiction. SMAD, Rev Eletrônica Saúde Mental Álcool Drog. 2021 abr.-jun.;17(2):64-71. doi: https://dx.doi.org/10.11606/issn.1806-6976.smad.2021.173585 


\title{
Prevalence of major depressive disorder in people with chemical addiction
}

\begin{abstract}
Objective: to verify the prevalence of Major Depressive Disorder in people with chemical dependence. Method: cross-sectional study with the application of the structured interview for the DSM-V disorders in 183 individuals admitted to therapeutic communities for chemical dependents, located in the south of Santa Catarina, during the year 2019. Results: All participants did not have a psychiatric consultation when entering the institution and the majority did not have a regular psychiatric consultation (94.5\%). It was found that 89 participants (55.3\%) had diagnostic criteria for Major Depressive Disorder and $59.1 \%$ of these had alcohol addiction $(p<0.028)$. Conclusion: the diagnosis of depressive disorder should be consolidated as an important variable for the effectiveness of treatment, since its prevalence is high and has repercussions on the quality of treatment and time of institutionalization.
\end{abstract}

Descriptors: Depressive Disorder, Major; Substance-Related Disorders; Epidemiology; Rehabilitation.

\section{Prevalencia del trastorno depressive major en personas con adicción}

Objectivo: verificar la prevalencia del transtorno depressivo major en personas con adicción. Metodo: estudio transversal con aplicación de la entrevista estructurada para los transtornos del DSM-V en 183 personas internadas en comunides de tratamiento de adicción en el departamento de Santa Catarina durante el ano de 2019. Resultados: la totalidad de los pacientes no realizaron cita con psiquiatra al ingressar en las instituiciones y la mayoria no realiza visitas regulares al psiquiatra $(94,5 \%)$. Se observó que 89 participantes $(55,3 \%)$ tenian critérios diagnósticos para depressión y $59,1 \%$ de ellos eram adictos al álcohol $(p<0,028)$. Conclusión: el diagnóstico del transtorno depressivo debe consolidarse como una variable importante para la eficácia del tratamiento, pues su prevalencia es elevada y pose repercursiones sobre la calidad del tratamiento y el tiempo de permanencia en las comunidades.

Descriptores: Trastorno Depresivo Mayor; Transtornos Relacionados con Sustancias; Epidemiologia; Rehabilitación. 


\section{Introdução}

A identificação do Transtorno Depressivo Maior (TDM) em pessoas com dependência química é importante tanto para o prognóstico quanto para o planejamento e desenvolvimento de condutas adequadas, pois sua concomitância pode modificar os sinais e sintomas próprios de cada comorbidade, elevando o número de intervenções terapêuticas inadequadas e ineficazes ${ }^{(1-2)}$.

Ainda que tenha havido um incremento na pesquisa sobre transtornos depressivos em pessoas com dependência química, no Brasil, são poucos os estudos que investigaram tal questão(1). Uma pesquisa, realizada no Estado do Rio Grande do Sul, verificou a frequência de comorbidades psiquiátricas em dependentes químicos e arrojou, entre seus resultados, que $16,1 \%$ da população selecionada preenchia critérios diagnósticos para TDM(3).

Acrescenta-se a este fenômeno o elevado número de indivíduos adictos subdiagnosticados com condições psicopatológicas, o que eleva o montante de prejuízos relacionados à doença de base, pois o sujeito com TDM, por exemplo, pode ficar mais limitado na busca de ajuda ou na compreensão de sua doença ${ }^{(2,4)}$. Com base na magnitude do fenômeno supracitado, faz-se necessária uma investigação da prevalência e características próprias dos pacientes com duplo diagnóstico (dependência química/ depressão) na região sul de Santa Catarina, pois o manejo incorreto destes indivíduos, além de promover a piora na qualidade de vida dos mesmos e seus familiares, gera um elevado custo social(5-6).

Igualmente, são necessários dados epidemiológicos confiáveis para respaldar o desenvolvimento e/ou a atualização de políticas públicas direcionadas à resolução deste fenômeno. Por sua vez, o objetivo deste estudo foi verificar a prevalência do TDM em pessoas com dependência química internadas em comunidades terapêuticas localizadas no sul de Santa Catarina.

\section{Método}

Realizou-se um estudo transversal em duas Comunidades Terapêuticas de Acolhimento a Dependentes Químicos (CTDQs), localizadas no sul de Santa Catarina, entre os meses de julho e dezembro de 2019. Por meio de cálculo amostral realizado na Plataforma (OpenEpi) para a obtenção do intervalo de confiança de $95 \%$, erro 1 -alpha de $5 \%$ e erro tipo II de $20 \%$, o número ideal de participantes foi de 183 participantes.

Palestrou-se, nas CTDQs, para um público de 702 indivíduos internos. Destes, 602 voluntariaram-se, sendo que 543 cumpriram os requisitos iniciais (tempo superior a 29 dias de internação, maiores de idade e menores a 60 anos, não terem diagnóstico prévio de esquizofrenia). Destes, foram sorteados, por aplicativo eletrônico, 183 participantes para a pesquisa.
O primeiro passo foi a coleta das variáveis individuais (não direcionada a verificar a dimensão psicológica dos participantes), que foi realizada por meio de questionário autoadministrado. $\mathrm{O}$ instrumento foi confeccionado pelos pesquisadores e manteve todos os preceitos éticos da declaração de Helsinki. Para garantir que ele fosse de fácil compreensão e entendimento dos participantes, executou-se um pré-teste com o grupo de pesquisa, comparando o mesmo a outros dois instrumentos de coleta de dados com objetivos e conteúdos similares.

Concomitantemente, realizou-se a Entrevista Clínica Estruturada para os Transtornos do DSM-5 (SCID-V-CV) ${ }^{(7)}$ por psicólogos especialistas em avaliação psicológica, com os itens E1 - E36: transtornos do uso de álcool e outras substâncias - Dependência ao Álcool e Outras Drogas para avaliar a variável "presença de dependência química". As questões foram direcionadas a aferir frequência de consumo, problemas associados, preocupação a respeito do uso por parte do círculo social proximal, prejuízo na execução de tarefas esperadas, tentativas malsucedidas de cessar ou reduzir o uso, sentimento de compulsão, entre outros ${ }^{(6)}$. Essa entrevista teve duração média de 30 minutos e foi realizada de maneira individual em sala fechada.

Prosseguiu-se a entrevista com o SCID-V-CV (itens E27/E34: transtornos do uso de álcool e outras substâncias - abstinência) com o objetivo de descartar um possível estado de intoxicação ou abstinência. Este procedimento teve como objetivo evitar o viés de confusão entre os sintomas negativos do humor decorrentes do estado de abstinência/desintoxicação com os de TDM. Os participantes identificados com estado de abstinência/ intoxicação não prosseguiram para os próximos passos do estudo.

Para evitar o viés de confusão entre os sintomas negativos de humor devido à privação de liberdade ou outra condição médica psiquiátrica que não seja o TDM, realizou-se a aplicação dos itens A45 - A64 do SCID-V-CV (transtorno distímico/transtorno de humor devido a uma condição médica geral). Os participantes identificados com estado de humor alterado devido a outras causas não prosseguiram para os próximos passos do estudo. Posteriormente, foram avaliadas as variáveis "prevalência do Transtorno Depressivo Maior" por meio do SCIDV-CV, itens A1 - A12 (TDM atual). Com essa entrevista, identificou-se a presença de depressão.

A análise dos achados foi realizada da seguinte forma: os dados obtidos nos questionários foram tabulados em um banco de dados do software estatístico SPSS (Statistical Package for Social Sciences), versão 21. A apresentação dos dados qualitativos foi realizada em números totais, porcentagem (\%), enquanto os dados quantitativos foram expressos por média e desvio-padrão e valores mínimo, máximo e amplitude. 
Para as comparações entre as variáveis sociais qualitativas, foi aplicado o teste qui-quadrado de Pearson para a determinação da frequência de ocorrência. Já para as variáveis quantitativas, foram realizados o teste t-Student para a comparação entre os pacientes com e sem TDM e a correlação de Pearson para verificar a associação entre as variáveis quantitativas. O nível de significância determinado foi de $p \leq 0,05$. O número total de participantes propiciou que as análises estatísticas tivessem o intervalo de confiança de $95 \%$ e o poder de estudo de $80 \%$.

Este projeto foi submetido à apreciação do Comitê de Ética em Pesquisa conforme a Resolução no 510/16, respeitando o anonimato dos participantes e a permissão da instituição em estudo. A coleta de dados foi realizada após a aprovação pelo Comitê de Ética em Pesquisa (CEP/UNISUL: 3.442.587 - CAAE:09833119.9.0000.5369).

\section{Resultados}

\section{Caracterização dos participantes}

As variáveis individuais e sociais dos participantes, referidas no questionário autoadministrado, estão descritas na Tabela 1, variáveis quantitativas, e Tabela 2 , respectivamente.

Tabela 1 - Variáveis quantitativas referidas no questionário autoadministrado relacionadas à internação $(n=183)$. Criciúma, SC, Brasil, 2019

\begin{tabular}{|c|c|c|c|c|c|}
\hline & Mínimo & Máximo & Amplitude & Média & Desvio-padrão \\
\hline Idade (anos) & 18 & 59 & 41 & 37,08 & 10,79 \\
\hline Tempo de internação (dias) & 30 & 271 & 259 & 87,78 & 57,60 \\
\hline Idade de início do uso (anos) & 10 & 27 & 17 & 14,03 & 2,23 \\
\hline Número de internações & 1 & 12 & 11 & 5,235 & 2,887 \\
\hline
\end{tabular}

Tabela 2 - Variáveis qualitativas referidas no questionário autoadministrado ( $n=183)$. Criciúma, SC, Brasil, 2019

\begin{tabular}{|c|c|c|}
\hline & $\mathrm{n}$ & $\%$ \\
\hline \multicolumn{3}{|l|}{ Religião } \\
\hline Agnóstico & 6 & $3,3 \%$ \\
\hline Ateu & 3 & $1,6 \%$ \\
\hline Católico & 151 & $82,5 \%$ \\
\hline $\begin{array}{l}\text { Protestante } \\
\text { evangélico }\end{array}$ & 23 & $12,6 \%$ \\
\hline \multicolumn{3}{|l|}{$\begin{array}{l}\text { Familiares de primeiro } \\
\text { grau com dependência } \\
\text { química }\end{array}$} \\
\hline Sim & 22 & $12,0 \%$ \\
\hline Não & 161 & $88,0 \%$ \\
\hline \multicolumn{3}{|l|}{$\begin{array}{l}\text { Consulta psiquiátrica ao } \\
\text { ingressar }\end{array}$} \\
\hline Não & 183 & $100,0 \%$ \\
\hline \multicolumn{3}{|l|}{$\begin{array}{l}\text { Consulta psiquiátrica } \\
\text { regular }\end{array}$} \\
\hline Sim & 10 & $5,5 \%$ \\
\hline Não & 173 & $94,5 \%$ \\
\hline \multicolumn{3}{|l|}{ Estado civil } \\
\hline Solteiro & 158 & $86,3 \%$ \\
\hline Casado & 16 & $8,7 \%$ \\
\hline União estável & 9 & $4,9 \%$ \\
\hline \multicolumn{3}{|l|}{ Escolaridade } \\
\hline Não alfabetizados & 4 & $2,2 \%$ \\
\hline Ensino Fundamental & 36 & $19,7 \%$ \\
\hline Ensino Médio & 137 & $74,9 \%$ \\
\hline Ensino Superior & 6 & $3,3 \%$ \\
\hline
\end{tabular}

Quando avaliada a associação entre a idade de início de uso de drogas e o número de internações, bem como a idade de início de uso de drogas e tempo de internação, não houve resultados estatisticamente significativos ( $r=$ $0,028, p=0,76$, e $r=-0,022, p=0,75$, respectivamente).

\section{Entrevistas estruturadas SCID-V-CV}

A avaliação do transtorno por uso de substâncias, analisada por meio do SCID-V-CV, demonstrou que 156 participantes $(84,7 \%)$ tiveram diagnóstico de transtorno por uso de álcool como problema principal, enquanto 16 $(8,6 \%)$ foram por dependência de crack e 12 (6,5\%) por adicção à cocaína.

Em relação à gravidade da dependência alcoólica, os dados demonstraram que 135 participantes $(87,1 \%)$ apresentaram transtorno por uso de álcool do tipo grave, 11 participantes $(6,7 \%)$ apresentaram dependência alcoólica moderada e dez indivíduos (6,2\%), transtorno por uso de álcool do tipo leve.

Algumas condições psicológicas podem cursar com sintomas similares ao TDM e, por vezes, se não diagnosticadas com precisão, podem ser confundidas com ele. Para evitar um viés de confusão, realizou-se a exclusão dessas patologias com o SCID-V-CV. Identificou-se que 21 participantes $(11,5 \%)$ possuíam transtorno distímico e sete $(3,8 \%)$ estavam em período de abstinência, ou seja, na maioria dos participantes da pesquisa $(84,7 \%)$, estavam descartados os possíveis vieses mencionados anteriormente.

Uma vez identificados os pacientes com diagnóstico de dependência química e que não se encontravam em 
período de abstinência ou com humor deprimido devido ao isolamento, procedeu-se à realização da entrevista, que avaliou a presença do TDM, na qual se constatou que 89 participantes $(55,3 \%)$ possuíam critérios diagnósticos para TDM e $72(44,7 \%)$ não confirmaram tal diagnóstico.
Destes, verificou-se que quatro participantes dependentes de crack, seis participantes dependentes de cocaína e 81 participantes adictos ao álcool possuíam diagnóstico de TDM, existindo associação estatística apenas entre o alcoolismo e a depressão, como demonstrado na Figura 1.

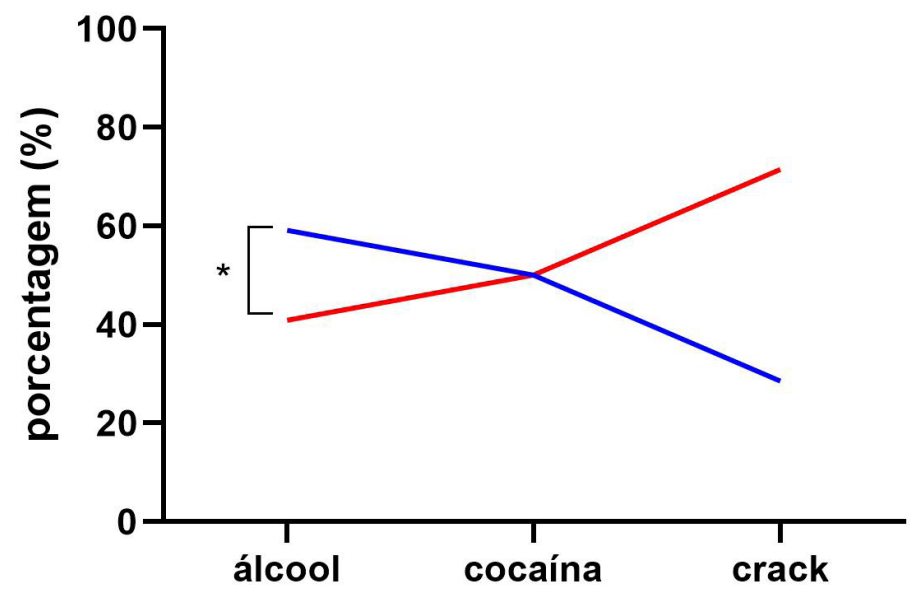

- presença de TDM

— ausência de TDM

${ }^{*} \mathrm{p}<0,028$ por teste qui-quadrado de Pearson, ${ }^{+} \mathrm{TDM}=$ Transtorno Depressivo Maior.

Figura 1 - Gráfico de porcentagem de pacientes com presença e ausência de TDM conforme o tipo de droga que foi motivo de internação. Criciúma, SC, Brasil, 2019

Dentre os participantes etilistas com TDM, $74(60,2 \%)$ preenchiam os critérios diagnósticos para alcoolismo grave e seis $(54,6 \%)$, moderado, demonstrando a associação entre a gravidade do alcoolismo e o $\operatorname{TDM}(p=0,003)$, segundo teste qui-quadrado de Pearson. Ao considerar os participantes que não possuem transtorno por abuso de álcool, nove $(52,9 \%)$ apresentam TDM.

Em relação às variáveis individuais e suas possíveis associações ao TDM, com exceção do "tempo de internação", não foram observadas correlações estatísticas, como se observa nas Tabelas 3 e 4.

Tabela 3 - Variáveis sociais quantitativas dos pacientes com presença e ausência de TDM*. Dados apresentados em média \pm desvio-padrão. Criciúma, SC, Brasil, 2019

\begin{tabular}{lccc}
\hline & $\begin{array}{c}\text { Presença } \\
\text { de TDM }\end{array}$ & $\begin{array}{c}\text { Ausência } \\
\text { de TDM }^{*}\end{array}$ & $\begin{array}{c}\text { valor } \\
\text { de }^{\dagger}\end{array}$ \\
\hline $\begin{array}{l}\text { Número de } \\
\text { internações }\end{array}$ & $5,19 \pm 2,86$ & $5,21 \pm 3,05$ & 0,970 \\
$\begin{array}{l}\text { Tempo de } \\
\text { internação (dias) }\end{array}$ & $87,72 \pm$ & $85,43 \pm$ & 0,027 \\
& 57,62 & 53,69 & \\
Idade atual (anos) & 10,82 & $37,89 \pm$ & 0,768 \\
& & 11,37 & \\
Idade de início de & $14 \pm 1,89$ & $14,08 \pm$ & 0,812 \\
uso (anos) & & 2,55 & \\
\hline
\end{tabular}

${ }^{*}$ TDM = Transtorno Depressivo Maior; ${ }^{\top}$ Teste Qui-Quadrado de Pearson
Tabela 4 - Variáveis sociais qualitativas dos pacientes com presença e ausência de TDM*. Dados apresentados em número (porcentagem). Criciúma, SC, Brasil, 2019

\begin{tabular}{|c|c|c|c|}
\hline & $\begin{array}{l}\text { Presença de } \\
\text { TDM }^{*}\end{array}$ & $\begin{array}{c}\text { Ausência de } \\
\text { TDM }^{*}\end{array}$ & $\begin{array}{l}\text { Valor } \\
\text { de } p^{\dagger}\end{array}$ \\
\hline Religião & & & 0,1407 \\
\hline Católico & $76(57,6 \%)$ & $56(42,4 \%)$ & \\
\hline $\begin{array}{l}\text { Protestante } \\
\text { evangélico }\end{array}$ & $8(40 \%)$ & $12(60 \%)$ & \\
\hline Agnóstico/ateu & $5(55,6 \%)$ & $4(44,4 \%)$ & \\
\hline Estado civil & & & 0,9406 \\
\hline Solteiro & $77(55,4 \%)$ & $62(44,6 \%)$ & \\
\hline $\begin{array}{l}\text { União estável/ } \\
\text { casamento }\end{array}$ & $12(54,6 \%)$ & $10(45,4 \%)$ & \\
\hline Escolaridade & & & 0,5074 \\
\hline Não alfabetizados & $2(50 \%)$ & $2(50 \%)$ & \\
\hline Ensino Fundamental & $13(44,8 \%)$ & $16(55,2 \%)$ & \\
\hline Ensino Médio & $72(58,5 \%)$ & $51(41,5 \%)$ & \\
\hline Ensino Superior & $2(40 \%)$ & $3(60 \%)$ & \\
\hline
\end{tabular}

${ }^{*}$ TDM $=$ Transtorno Depressivo Maior; ${ }^{+}$Teste Qui-Quadrado de Pearson

\section{Discussão}

A depressão, em indivíduos com dependência química, pode comprometer não apenas o tratamento da patologia de 
base, como também potencializar os sintomas depressivos caso não haja um diagnóstico e acompanhamento psicológico adequado ${ }^{(8-10)}$. Este estudo teve como hipótese que indivíduos internados em clínicas de reabilitação possuíam TDM e, por sua vez, sofriam com subdiagnósticos.

A compreensão desta comorbidade dual e sua representação em contextos de isolamento social ainda é um campo pouco investigado e permite inferir como e em que bases ela se constrói(1-3). Tais questões, além de constituírem interrogantes acadêmicas, são importantes pontos de partida para a construção de metodologias de enfrentamento mais funcionais.

Entre diferentes autores, pode-se encontrar um nível de similaridade para algumas variáveis que descrevem o perfil de pacientes residentes de centros de reabilitação para a dependência química(2,11-12). Os dados sociodemográficos da população deste estudo são semelhantes aos encontrados por grande parte dos trabalhos analisados, como média de idade de 37,8 anos, solteiros $(86,3 \%)$, religiosos $(98,4 \%)$ e com Ensino Médio completo (74,9\%).

A categoria de estado civil "solteiro" pode ser interpretada por meio de dois ângulos: o primeiro como base para a dependência química devido a uma possível perda do fator de proteção exercida pelo laço familiar/ conjugal ou o segundo como consequência das atitudes e comportamentos nocivos que esta doença reflete, produzindo um afastamento das pessoas de seu círculo social(2,13-14).

A média de idade entre os participantes foi ao encontro da de outros estudos ${ }^{(1,3)}$. Grande parte da população começou o uso de substâncias psicotrópicas na adolescência (média $=14,03$ anos) e possui tempo de consumo de drogas superior a 30 anos. A droga de início de uso, na maioria dos casos, foi o álcool, possivelmente pelo fato de ser uma substância lícita e de baixo custo, assim como bem aceita socialmente.

Ao contrário de outros trabalhos, o uso de substâncias desde a tenra idade não pareceu prejudicar a realização dos estudos até o grau médio (74,3\%). Entretanto, parece ser um fator que contribui para a não realização de curso superior $(96,7 \%)$. Por outro lado, estudos populacionais sugerem que pessoas com baixa escolaridade ou não alfabetizadas apresentam maior prevalência de psicopatologias, entre elas, a dependência química(13,15). Assim como um estudo realizado no Estado do Rio Grande do Sul demonstrou que a frequência de psicopatologias em indivíduos com formação superior foi baixa em relação aos não escolarizados, sugerindo que a educação superior seria um fator de proteção contra a dependência química(1).

No Brasil, a idade média de conclusão do Ensino Médio é de $17 \pm 1$ anos. Por outro lado, neste estudo, a média de idade de início de uso de drogas foi de 14 anos. Ao analisar tais achados, pode-se conjecturar que a frequência de consumo de substâncias psicoativas aconteceu de maneira gradual, assim como outros fatores, como a pressão por parte dos pais (para não abandonar a escola), das instituições (ameaças de contatar conselhos tutelares) e/ou subjetivas (medo, ausência de adicção, baixo poder de compra das drogas), contribuíram para o não abandono dos estudos. Todavia, uma vez maiores de idade e com três ou mais anos de uso, os fatores de proteção supracitados perderiam força, o que, por sua vez, auxiliaria na não realização de cursos superiores.

Uma vez que estes sujeitos se encontram em vulnerabilidade emocional e desestabilidade social, o diagnóstico preciso do TDM ao ingressar na instituição e o acompanhamento psicológico/psiquiátrico regular são essenciais para o manejo correto da depressão no sujeito com dependência química, pois, uma vez não tratada a comorbidade, as probabilidades da não resolução da doença de base elevam-se significativamente(16-18). Os dados obtidos demonstraram que nenhum paciente das duas clínicas recebeu consulta psiquiátrica ao ingressar e que apenas 5,5\% frequentavam regularmente consultórios de Psiquiatria e Psicologia. Tal informação leva a indagar que, se os pacientes estão internados pela dependência química e não recebem diagnóstico de outras psicopatias durante o período de internação, possivelmente haja subdiagnósticos e, consequentemente, falhas no tratamento.

Como agravante desta ausência de longitudinalidade e integralidade do cuidado, identificou-se que mais da metade dos pacientes internados possui critérios segundo o instrumento SCID-V-CV para TDM (55,3\%), uma vez que se excluíram as patologias que poderiam interferir no diagnóstico por apresentarem padrões de sintomas similares, como: abstinência $(3,8 \%)$ e a tristeza devido ao isolamento e à privação de liberdade $(11,5 \%)$.

Em outros estudos, os resultados demonstraram que a média de TDM em dependentes químicos varia entre 18 e $26 \%(4,6)$, ou seja, os participantes desta pesquisa possuíam o dobro de prevalência (55,3\%). Este dado pode ser interpretado por meio de dois ângulos principais: a) o instrumento utilizado possui maior sensibilidade diagnóstica e, com isso, maior poder de detecção; b) a população analisada vive privada de liberdade, não possuindo interferência de fatores externos, o que poderia concentrar os sintomas depressivos. Em suma, internos de CTDQs da região sul catarinense possuem maior prevalência de TDM em comparação aos de outros trabalhos nacionais e internacionais.

Ainda, a prevalência do transtorno depressivo foi maior em indivíduos dependentes de álcool (59,1\%) em relação aos dependentes de outras substâncias, sendo que a dependência alcoólica guardou significância estatística com o diagnóstico de depressão $(p=0,028)$. Além disso, também foi constatada significância em relação à gravidade da dependência alcoólica, sendo que os usuários com dependência grave $(60,2 \%)$ possuem 
maior índice de TDM. Por outro lado, usuários com dependência alcoólica leve não guardaram relação com o diagnóstico de TDM.

Da mesma forma, um trabalho descreveu que o abuso do álcool guarda associação com a depressão e relatou que esta informação pode ter origem no fato de que há um conglomerado de problemas associados ao uso abusivo de álcool (efeitos adversos à saúde, problemas no trabalho, nas relações familiares, na convivência em círculos sociais que não utilizam drogas) e que o abuso de álcool pode contribuir para a ocorrência da depressão(1,3-19-20).

Alguns autores acrescentaram que, quando não tratada, a presença de psicopatologias em pacientes com dependência química, entre elas, o TDM, pode levar a lapsos ou recaídas em relação ao uso de substância. Esta afirmação parece corroborar os dados encontrados neste estudo, pois, ao observar a variável "número de internações", identificou-se que a média foi de 5,23 \pm 2,887 , ou seja, na maioria dos casos, houve falha no tratamento da primeira internação e a grande maioria dos pacientes é redicivante.

Observou-se que o grupo dos participantes com depressão permaneceu menos tempo internado em relação aos não depressivos $(p=0,027)$, dado que pode indicar que o fato de o sujeito conviver com depressão influi diretamente no resultado do tratamento, pois os sujeitos com essa condição abandonam com maior rapidez os programas de reabilitação, comprometendo o objetivo das CTDQs em melhorar o quadro da dependência química. Uma vez que os sujeitos não tratados readquirem sua liberdade com uma condição psicopatológica marcante como a depressão, podem experimentar sentimento de frustração por não conseguirem terminar o tratamento e, com isso, favorecer a reutilização de drogas como meio de alívio das dores emocionais ${ }^{(18,20-23)}$.

Por outro ângulo, os programas dirigidos pelos centros de reabilitação no sul de Santa Catarina têm duração de 360 dias, sendo que, neste estudo, se observou que o número médio de dias internados dos avaliados foi de $87,78 \pm 57.6$.

Por tratar-se de um estudo transversal, os achados correspondem a um recorte temporal específico, constituindo uma limitação deste trabalho, pois não houve a possibilidade de verificação da relação de causalidade entre as variáveis depressão e dependência química. Assim, sugere-se a realização de pesquisas que abranjam um período maior de seguimento, possibilitando uma maior compreensão desta problemática.

\section{Conclusão}

Os achados demonstraram que a presença de depressão nos sujeitos com dependência química é elevada. Assim sendo, faz-se essencial o diagnóstico/triagem desta comorbidade no sujeito que ingressa em CTDQs. Além do mais, a coocorrência destas psicopatologias diminui o tempo de internação (abandono do processo), da mesma forma que pode contribuir para a não eficácia do tratamento. É fato que ambos os quadros psicopatológicos se potencializam quando em comorbidade, gerando custos de reinternação para os adictos e entidades públicas financiadoras, assim como produzindo ou aumentando a carga de sofrimento psíquico.

Por fim, tais achados podem contribuir para o desenvolvimento de estratégias terapêuticas que, levando em consideração a possibilidade de depressão em indivíduos diagnosticados com dependência química, sejam mais assertivas e eficazes. Nesse sentido, estudos que visem a abordar esta problemática de forma mais ampla, envolvendo um número maior de pacientes e com maior diversidade de locais, poderão contribuir, de forma substancial, para a elaboração das possíveis estratégias a serem tomadas no que diz respeito ao tratamento de pessoas que sofram de dependência química.

\section{Referências}

1. Hess ARB, Almeida RMM de, Moraes AL. Comorbidity of psychiatric disorders with drug addiction: preliminary results. Estud Psicol. [Internet] 2012 [cited Nov 9, 2020];17(1):171-82. Available from: http://www.redalyc. org/articulo.oa?id=26122929021

2. Santos IMC, Santiago TRS, Oliveira JRV, Lima DE, Melo MRA. Psychological Evaluation with Users of Psychoactive Substances (SPA): a Systematic Review of Literature. Psi Unisc. 2018;2(1):48-60. doi: https:// dx.doi.org/10.17058/psiunisc.v2i2.10837

3. Scheffer M, Pasa GG, Almeida MMR. Alcohol, Cocaine, and Crack Dependence and Psychiatric Disorders. Psicol Teor Pesq. 2010;26(3):533-41. doi: https://doi. org/10.1590/S0102-37722010000300016

4. Heyman GM. Do addicts have free will? An empirical approach to a vexing question. Addict Behaviors Reports. 2017;5:85-93. doi: http://dx.doi.org/10.1016/j. abrep.2017.02.001

5. Crauss RMG, Abaid JLW. Chemical addiction and the hospital detoxification treatment in the speech of the users. Contextos Clín. 2012;5(1):62-72. doi: http:// dx.doi.org/10.4013/ctc.2012.51.07

6. Migott AMB. Chemical addiction: a biological, psychological or social problem?. Cad Saúde Pública. 2008;24(3):710-1. doi: https://doi.org/10.1590/ S0102-311X2008000300027

7. Hasin DS, O'Brien CP, Auriacaombe M, Borges G, Bucholz K, Budney A, et al. DSM-5 Criteria for Substance Use Disorders: Recommendations and Rationale. Am J Psychiatry. 2014;170(8):834-51. doi: http://doi. org/10.1176/appi.ajp.2013.12060782

8. Hoffmann NG, Kopak AM. How well do the DSM-5 alcohol use disorder designations map to the ICD-10 
disorders? Alcohol Clin Experiment Res. 2015;39(4):697701. doi: https://doi.org/10.1111/acer.12685

9. Souza AM. Compreensões psicológicas sobre a dependência química. Psicologia.pt. 2017 [cited Nov 9, 2020];1(1)1-17. Available from: https://www.psicologia. pt/artigos/textos/TL0425.pdf

10. Sebena R, Ansari W, Stock C, Orosova O, Mikolajczyk RT. Are perceived stress, depressive symptoms and religiosity associated with alcohol consumption? A survey of freshmen university students across five European countries. Subst Abus Treat Prev Policy. [Internet]. 2012 [cited Nov 9, 2020];7(1):1-10. Available from: http:// www.substanceabusepolicy.com/content/7/1/21

11. Cantão L BN. Suicidal behavior among drug addicts. REBEn. 2016;69(2):389-96. doi: http://dx.doi. org/10.1590/0034-7167.2016690224i

12. Ciribelli EB, Luiz AMAG, Gorayeb R, Domingos $N A M$, Marques $A B$ Filho. Intervention in waiting room of outpatient clinic addiction: characterization and evaluation of the effects. Temas Psicol. [Internet]. 2016 [cited Nov 9, 2020];16(1):10718. Available from: http://pepsic.bvsalud.org/scielo. php?script=sci_arttext\&pid=S1413-389X2008000100009 13. Bastos FI. Epidemiology about chemical dependence in Brasil. NUTE-UFSC. [Internet] 2012 [cited Nov 9, 2020]; 1(1):107-13. Available from: http://aberta.senad.gov.br/ 14. Belsky DW, Moffitt, TE, Corcoran DL, Domingue B, Harrington $\mathrm{H}$, Hogan $\mathrm{S}$, et al. The genetics of success: How single-nucleotide polymorphisms associated with educational attainment relate to life-course development. Psychol Sci. 2016;1(27):957-72. doi: https://doi. org/10.1177/0956797616643070

15. Presidencia de la Republica del Uruguay. VI Questionnaire National residences on drug use. JND. [Internet] 2016 [cited Nov 9, 2020];1(1):9-115. Available from: http://www.infodrogas.gub.uy/images/stories/ pdf/201609_VI_encuesta_hogares_OUD_ultima_rev.pdf 16. Quadros LCM, Laura HC, Quevedo LA, Gigante DP. Effects of social mobility on adult mental health:a systematic review of the literature. Ciênc Saúde Coletiva. 2016;21(2):443-8. doi: http://doi. org/10.1590/1413-81232015212.17942014

17. Sadock BJ, Sadock VA, Ruíz P. Compêndio de psiquiatria: Ciência do comportamento e psiquiatria clínica. Porto Alegre, RS: Artmed; 2016. 1490 p

18. Gonçalves JS, Silva LL, Abdala GA, Meira MDD, Santos ACM, Silva MFF. Religiosity and common mental disorders in adults. Rev Enferm UFPE online. 2017; 11 (Supl.4):1708-15.doi: 10.5205/ relou.10438-93070-1-RV.1104sup201713

19. Volkow ND, Koob GF, McLellan T. Neurobiologic advances from the brain disease model of addiction. $\mathrm{N}$ Engl J Med. 2016;374:363-71. doi: http://doi.org/10.1056/ NEJMra1511480

20. Volkow ND, Koob G. Brain disease model of addiction: why is it so controversial? Lancet Psychiatry. 2015;2:6779. doi: https://doi.org/10.1016/S2215-0366(15)00236-9 21. Litten RZ, Ryan ML, Falk DE, Reilly M, Fertig JB, Koob GF. Heterogeneity of alcohol use disorder: understanding mechanisms to advance personalized treatment. Alcohol Clin Exp Res. 2015;39:579-84. doi: https://doi. org/10.1111/acer.12669

22. Lemieux $A$, al'Absi $M$. Stress psychobiology in the context of addiction medicine: from drugs of abuse to behavioral addictions. Prog Brain Res. 2016;223:43-62. doi: https://doi.org/10.1016/bs.pbr.2015.08.001

23. Kwako LE, Momenan R, Litten RZ, Koob GF, Goldman D. Addictions neuroclinical assessment: a neuroscience-based framework for addictive disorders. Biol Psychiatry. 2015; 1(1): 1-9. doi: http://doi.org/10.1016/j.biopsych.2015. 10.024

\section{Contribuição dos autores}

Concepção e desenho da pesquisa: Pablo Michel Barcelos Pereira, Rafael Mariano de Bitencourt. Obtenção de dados: Pablo Michel Barcelos Pereira. Análise e interpretação dos dados: Pablo Michel Barcelos Pereira, Rafael Mariano de Bitencourt. Análise estatística: Pablo Michel Barcelos Pereira. Redação do manuscrito: Pablo Michel Barcelos Pereira Revisão crítica do manuscrito quanto ao conteúdo intelectual importante: Pablo Michel Barcelos Pereira, Rafael Mariano de Bitencourt.

Todos os autores aprovaram a versão final do texto.

Conflito de interesse: os autores declararam que não há conflito de interesse. 